\title{
Power Management over Co-channel Femtocells in LTE-A Systems
}

\author{
Christos Bouras $^{* \dagger}$, Georgios Diles ${ }^{\dagger}$, Vasileios Kokkinos* ${ }^{* \dagger}$, Andreas Papazois*† \\ *Computer Technology Institute and Press "Diophantus", Patras, Greece \\ ${ }^{\dagger}$ Computer Engineering and Informatics Dept., University of Patras, Greece \\ bouras@cti.gr, diles@ceid.upatras.gr, kokkinos@cti.gr, papazois@ceid.upatras.gr
}

\begin{abstract}
The use of femtocells has been an attractive solution since it achieves better coverage and capacity and low cost for deployment and maintenance. However, their performance can be compromised by the cross-tier interference with existing macrocell infrastructure or between adjacent femtocells, especially in the case of co-channel deployment. One way to address this, is adjusting the transmit power of every femto base station with respect for overall performance. To this direction, we have implemented a framework that simulates femtocell overlays over LTE-Advanced (LTE-A) macrocellular systems. The framework allows power management over user-defined femtocell deployment, deciding their power levels according to three different power schemes. The resulting throughput is presented for every point of the macrocell in a user-friendly GUI. In this paper, we present the design of this framework and discuss the results.
\end{abstract}

\section{INTRODUCTION}

Femtocells, also referred to as femto or Femto Base Stations (FBSs) or Home Node-Bs (HNBs), are short-range, userdeployed base stations that improve locally the capacity and coverage of the mobile network, while leading to high spectral efficiency. Achieving the latter, though, without creating significant interference to the existing macrocell or adjacent femtocell services is a main challenge of femto technology, especially for co-channel Closed Subscriber Group (CSG) femtocell deployment, the most attractive deployment regarding spectral efficiency. One way to address this, is adjusting the power transmission levels of FBSs, in order to minimize intra-cell and inter-cell interference.

A major portion of the existing literature has investigated the interference and power management issues of femtocell deployment. Simulation results of interference impact on downlink and uplink throughputs are presented in [1] and [2], for co-channel femtocells. A power control method is also introduced in [2], that ensures a constant femtocell radius. FBS pilot power is adjusted in [3], based on the number of handover events and the indoor users, while a distributed power management algorithm in [4] uses soft constraints on target Signal to Interference plus Noise Ratio (SINR) values. Regarding simulation tools, open source frameworks in [5] and the Vienna simulators[6] provide solid system level simulations for LTE networks while the work in [7] focus on femtocells.

Although the above works provide a solid description of femtocell interference and power management issues, they do not offer a user-friendly graphical tool designed to reproduce and calculate the optimal femtocell transmission parameters, via a graphical representation of the entire macrocell site for a highly customizable network configuration. In this paper we extend the simulation framework presented in [8], where the throughput is calculated in every point of a macro site portrayed as a 2-D plane, for custom femtocell deployment. This tool takes as inputs the number and position of the FBSs, the number of femto and macro-users and the density of the custom urban environment.

The contribution of this paper is the inclusion of power management over the FBSs. The pilot and the operational transmission power of every FBS are calculated according to the power scheme selected of the three available choices: The default fixed power transmission for every FBS, the individual power configuration to achieve constant coverage femtocell radius, and the iterative scheme focusing on achieving target SINR values when possible. The resulting performance is calculated and presented graphically, for every type of user. We also added flexibility, allowing the user to customize every aspect of the network configuration, such as desired femto range or penetration loss values, increasing the application range of the tool. Finally, we accelerated the simulation process by approximately $30 \%$. The above mechanism covers some general power control approaches providing an insight for the boost of performance the latter achieves for userdefined network topologies.

The rest of the paper is organized as follows. In Section II we provide a description of the simulator model and configuration. In Section III specific experimental results are given and a evaluation is taking place. Finally, we conclude our paper and suggest future enhancements in Section IV.

\section{SySTEM MODEL AND CONFIGURATION}

Since femtocells are to be deployed by the often nonqualified average user, the simulation environment needs to be user friendly and built in a structured way that allows easy future expansion. The framework proposed in this paper follows the above rules. The user interacts through a simple graphical interface and he is asked to provide the custom parameters. The required inputs depend on the qualification of the user. A typical instance of the above interface is illustrated in Fig. 1, displaying the first stages of the process, that include network parameters and topology configuration. 
The inputs trigger the necessary calculations of the FBSs power transmission according to the selected power control algorithm. When the process stops, the map is coloured based on the estimated throughput. This way the user gains a userfriendly, comprehensive graphical overview of the resulting performance as illustrated in the examples of Section III. Detailed information for the results can be obtained for any point of the topology, by clicking anywhere in the map.

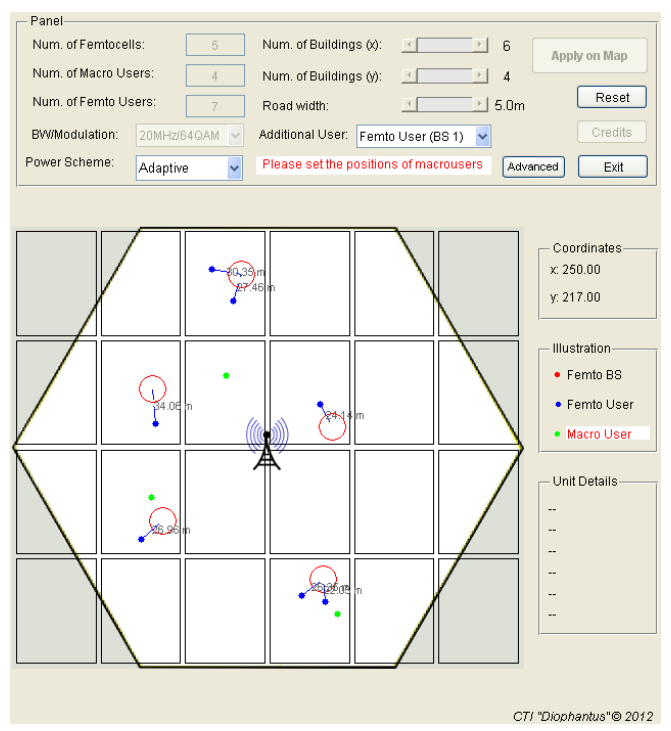

Fig. 1: Typical instance of the user interface.

To calculate the final throughput, SINR and the capacity are evaluated, taking into consideration the path loss and propagation models as they are described in [9], [10]. The power control schemes integrated to determine the transmission power of each FBSs are the following. The first one is the simplest of all, assigning a fixed value for every FBS. The second method is introduced in [2], and ensures a constant coverage femtocell radius $r$, by setting FBSs power value equal to the power received from the MBS at a target femtocell radius $r$. Specifically:

$$
P_{f}=\min \left(P_{m}+G_{\theta}-P L_{m}(d)+P L_{f}(r), P_{\max }\right)
$$

where $P L_{f}(r)$ is path loss at the radius $r, P L_{m}(d)$ the macrocell path loss at distance $d, P_{m}$ is the MBS transmit power and $G_{\theta}$ is the antenna gain.

The third approach readjusts FBSs transmission power periodically in order to achieve the desired SINR value at a user-specified range when that is feasible. If $S I N R_{t}$ and $S I N R_{c}$ denote the target and current SINR respectively, the converging power control algorithm is given by[4]:

$$
P(k+1)=\frac{S I N R_{t}}{S I N R_{c}} P(k)
$$

$P(k)$ denotes the power level of FBS at the $k$ th iteration. The algorithm either converges adequately or reaches the maximum allowed values.

\begin{tabular}{|c|c|c|c|c|c|}
\hline Parameter & \multicolumn{5}{|c|}{ Value } \\
\hline Mecrocell Radius $\left(R_{m}\right)$ & \multicolumn{5}{|c|}{$250 \mathrm{~m}$} \\
\hline Macro BS TX Power & \multicolumn{5}{|c|}{$46 \mathrm{dBm}$} \\
\hline Carrier frequency & \multicolumn{5}{|c|}{$2 \mathrm{GHz}$} \\
\hline FBS default / max TX Power & \multicolumn{5}{|c|}{$18 / 20 \mathrm{dBm}$} \\
\hline Outdoor/Indoor Walls Loss & \multicolumn{5}{|c|}{$15 / 7 \mathrm{~dB}$} \\
\hline Bandwidth $(\mathrm{MHz})$ & \begin{tabular}{l|l}
20 & 15 \\
\end{tabular} & 10 & 5 & 3 & 1.4 \\
\hline Modulation Mode & 64QAM & \multicolumn{2}{|c|}{ 16QAM } & \multicolumn{2}{|c|}{ QPSK } \\
\hline Subcarrier Spacing & \multicolumn{5}{|c|}{$15 \mathrm{kHz}$} \\
\hline
\end{tabular}

TABLE I: Simulation Parameters

Table I summarizes the default values used during the simulation, though an experienced user is encouraged to change them desirably. The network configuration considered for the simulator is urban area environment though the density of this environment is subject to the user preferences.

\section{Simulation RESUlts AND EVALUATION}

In Fig. 2 an example of the simulator results is illustrated. The throughput of a macro user when fixed pilot power of FBSs is applied(left), is compared with the one achieved when constant radius of FBS coverage power scheme(right) is chosen. The beneficial suppression of femtocell power in respect for macrocell performance is shown, especially for the distant indoor areas due to increased path loss.

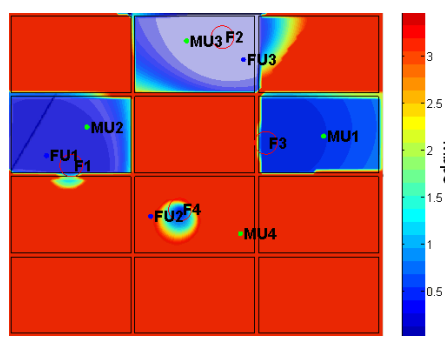

(a)

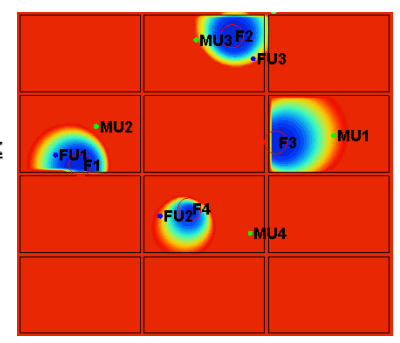

(b)
Fig. 2: Throughput of a macro-user in the presence of femtocells for: (a) fixed power, (b) constant femtocell radius power.

An example of the periodically adaptive power control scheme is demonstrated in Fig. 3. The progressive adjustment of FBSs power levels is displayed through the resulting throughput of a femto-user for every successive iteration. Since the initial configuration is proven to be unsuited for the FBSs, due to the increased number of users for the first example (Fig. 3a), and the distant femtocell placement (Fig. 3b), the scheme is activated. At the third iteration, the algorithm converges.

A comparison of the different power schemes is demonstrated in Fig. 4. The resulting throughput is displayed for an indoor macro user that moves away from the femto base station and towards the distant $(>200 \mathrm{~m})$ MBS. Power management may achieve up to 17 times better throughput than the fixed power case. Finally, the average FBSs transmit power of 10 randomly located femtocells is portrayed in Fig. 5. The energy 


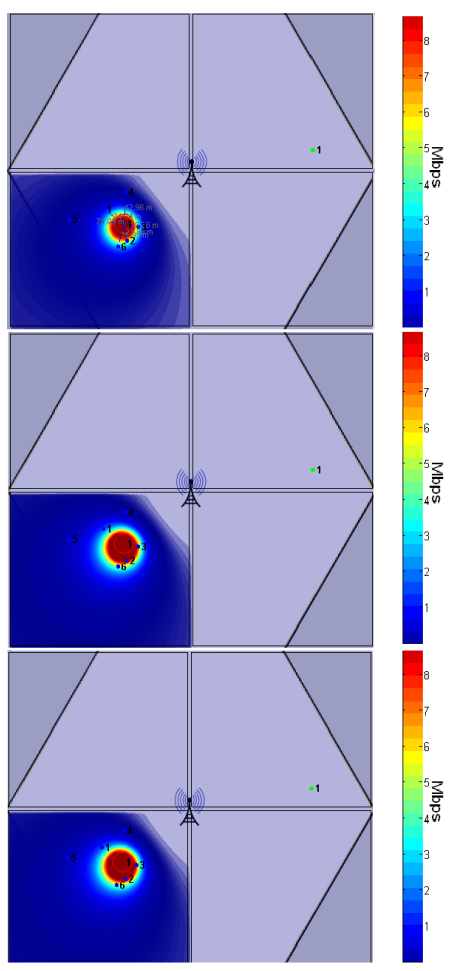

(a)

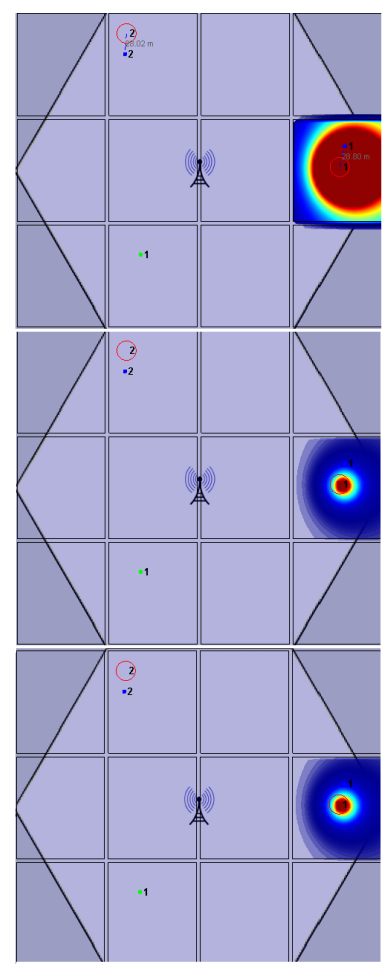

(b)
Fig. 3: Throughput of a femto-user for the successive adjustments of femto power levels for: (a) many femto-users, (b) a distant FBS.

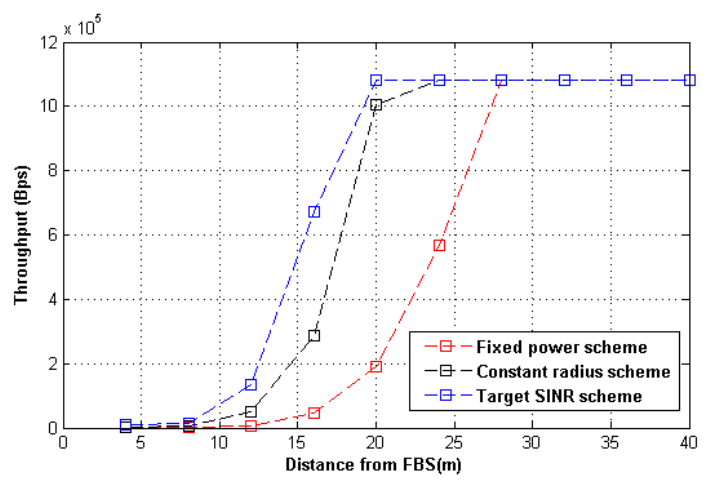

Fig. 4: Throughput for a macro-user near a FBS moving towards the MBS for the three power configurations.

savings are evident, reaching approximately $70 \%$ decrease for the two adaptive schemes.The results of the comparison though, vary largely depending on the custom configuration. Thus, the above mostly demonstrate the capabilities of the simulator, and secondly present a general behaviour of femtocells deployments, using typical configurations.

\section{COnClusions \& Future Work}

In this paper, we presented a simulation tool with power control capabilities over fully customized co-channel femtocells deployments. The simulator offers an insight of the result-

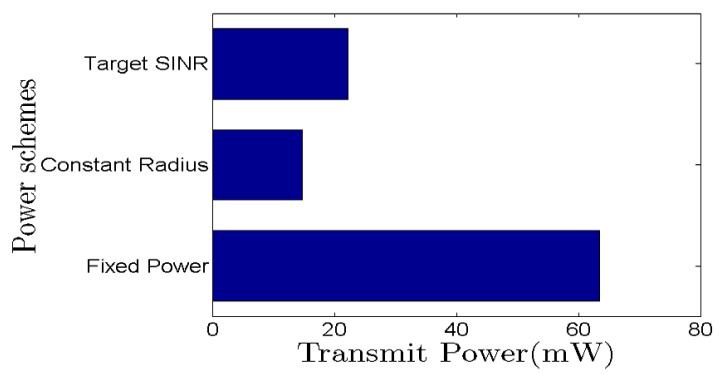

Fig. 5: Average FBS transmit power of randomly located femtocells.

ing network performance when utilizing power management. Three different power control schemes were integrated to cover the most general approaches, though further inclusions are possible. Other options to enhance the simulator is location management of the femtocells or the integration of radio allocation schemes such as fractional frequency reuse.The code of the simulator is available online for usage and possible expansion [11].

\section{REFERENCES}

[1] A. Alexiou, C. Bouras, V. Kokkinos, K. Kontodimas, and A. Papazois, "Interference behavior of integrated femto and macrocell environments," in IFIP Wireless Days 2011 (WD'11), 2011.

[2] H. Claussen, "Performance of macro- and co-channel femtocells in a hierarchical cell structure," in IEEE 18th International Symposium on Personal, Indoor and Mobile Radio Communications 2007 (PIMRC'07), 2007.

[3] H. Claussen, L. Ho, and L. Samuel, "Self-optimization of coverage for femtocell deployments," in Wireless Telecommunications Symposium 2008 (WTS'08), 2008, pp. 278-285.

[4] M. Xiao, N. Shroff, and E. Chong, "Utility-based power control in cellular wireless systems," in 12th IEEE Annual Joint Conference of the IEEE Computer and Communications Societies 2001, (INFOCOM'01.), vol. 1, 2001, pp. 412-421 vol.1.

[5] N. Baldo, M. Miozzo, M. Requena-Esteso, and J. Nin-Guerrero, "An open source product-oriented LTE network simulator based on ns-3," in 14th ACM international conference on Modeling, analysis and simulation of wireless and mobile systems, ser. MSWiM '11. New York, NY, USA: ACM, 2011, pp. 293-298. [Online]. Available: http://doi.acm.org/10.1145/2068897.2068948

[6] M. S. S. S. M. W. M. R. C. Mehlfhrer, J. Colom Ikuno, "The vienna LTE simulators - enabling reproducibility in wireless communications research," EURASIP Journal on Advances in Signal Processing, vol. 2011, no. 1 - 13, 2011.

[7] F. Capozzi, G. Piro, L. A. Grieco, G. Boggia, and P. Camarda, "A system-level simulation framework for LTE femtocells," in 5th International ICST Conference on Simulation Tools and Techniques, ser. SIMUTOOLS '12, ICST, Brussels, Belgium, 2012, pp. 211-213. [Online]. Available: http://dl.acm.org/citation.cfm?id=2263019.2263049

[8] C. Bouras, V. Kokkinos, K. Kontodimas, and A. Papazois, "A simulation framework for LTE-A systems with femtocell overlays," in 15th ACM international conference on Modeling, analysis and simulation of wireless and mobile systems (MSWiM'12), 2012, (to appear).

[9] H. Lei, L. Zhang, X. Zhang, and D. Yang, "A novel multi-cell ofdma system structure using fractional frequency reuse," in IEEE 18th International Symposium on Personal, Indoor and Mobile Radio Communications 2007 (PIMRC'07), 2007.

[10] 3GPP TR 36.814 V9.0.0, "Evolved Universal Terrestrial Radio Access (E-UTRA); Further advancements for E-UTRA physical layer aspects (Release 9)," 3rd Generation Partnership Project, Tech. Rep., 2010.

[11] "Femtocell Power Management Interference Simulator (FePIS)," http://ru6.cti.gr/ru6/fepis.zip. 